\title{
Cánones por uso de la Infraestructura Ferroviaria Española
}

\author{
Juan B. Font Torres \\ Máster en Infraestructuras del Transporte, Territorio y Urbanismo \\ Escuela Técnica Superior de Ingenieros de Caminos, Canales y Puertos \\ Doctorando en Infraestructuras de Transporte y Territorio \\ Universidad Politécnica de Valencia
}

\section{RESUMEN}

La Unión Europea adoptó una serie de directivas destinadas a revitalizar el sector ferroviario con el fin de convertir el ferrocarril en un modo de transporte competitivo. Estas Directivas se agruparon en los siguientes paquetes:

- La separación de actividades de administración de la infraestructura de las actividades propias de la explotación.

- La apertura del transporte ferroviario en régimen de libre competencia así como la asignación al administrador de infraestructuras ferroviarias de las funciones necesarias para la prestación de servicios en cuanto a transporte de viajeros como de mercancías.

Esto quedó plasmado en una serie de marcos tarifarios, en los cuales cada país creó unas reglas específicas de fijación de cánones o delegando su creación en el administrador de infraestructuras ferroviarias correspondiente. Esta aparición de este concepto de tarificación por uso de la infraestructura está unido a los procesos de liberalización del sector ferroviario. Como resultado ha dado lugar a tarifas muy diversas, no sólo bajo el aspecto económico sino también bajo el aspecto de nomenclatura a la hora de definir las mismas, obtenido como resultado de la inexistencia de una pauta detallada de cómo deberían fijarse los mismos. Estos cánones, aunque están regulados por la correspondiente Directiva Europea, son establecidos según la normativa del país, lo que da origen a una diversidad de los mismos. Ante esta diversidad de cánones, en cuanto a definición como a las fórmulas aplicadas para su obtención, resulta difícil conseguir que el transporte ferroviario sea competitivo frente a otros medios de transporte como pretenden las correspondientes Directivas Europeas.

La apertura del transporte ferroviario en régimen de libre competencia obliga a las empresas ferroviarias, para poder operar por la red ferroviaria europea, cumplir una serie de requisitos legales, técnicos y económicos, que quedan reflejados en una serie de cánones a abonar a los administradores de infraestructura ferroviaria de los países por donde circula un transporte ferroviario.

Tras analizar los cánones que dan acceso a la Red Ferroviaria de Interés General de ADIF éstos se deberían transformar en "precios" de forma que fuese más fácil y directa su adecuación a las condiciones del mercado existente. Además de los cánones por utilizar la infraestructura, debería existir una serie de bonificaciones e incentivos de dichos cánones por mayor aprovechamiento de la misma, como es el aumento del número de circulaciones, reduciendo la componente del canon tráfico a medida que sube el tráfico total de la línea con el mejor aprovechamiento de la misma. 


\section{LA INFRAESTRUCTURA FERROVIARIA EN ESPAÑA.}

En España la infraestructura ferroviaria está gestionada por ADIF (Administrador de Infraestructuras Ferroviarias). Se trata de una entidad pública empresarial, organismo público adscrito al Ministerio de Fomento, con personalidad jurídica propia, plena capacidad de obrar para el cumplimiento de sus fines y patrimonio propio.

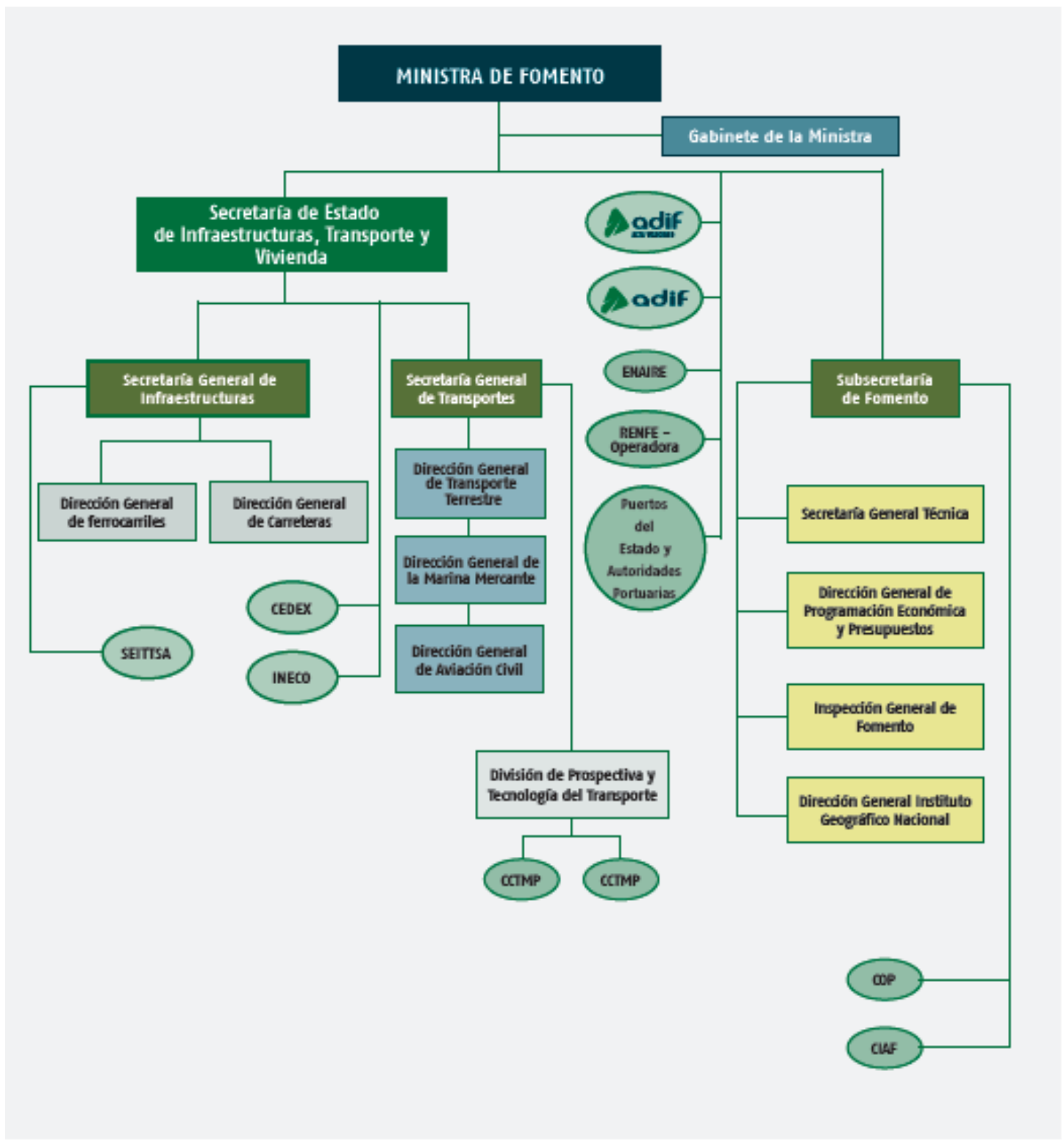

Figura 1 - Organigrama del Ministerio de Fomento.

Se rige por lo establecido en la Ley del Sector Ferroviario, en la Ley de Organización y Funcionamiento de la Administración General del Estado, LOFAGE, en las normas de desarrollo de ambas, en su propio Estatuto y en la legislación presupuestaria. En el ejercicio de sus funciones ADIF actúa con autonomía de gestión, dentro de los límites establecidos por su Estatuto y teniendo en cuenta, en todo caso, la garantía del interés público, la satisfacción de las necesidades sociales, la seguridad de los usuarios y la eficacia global del sistema ferroviario y los principios de transparencia, no discriminación, imparcialidad e independencia de cualquier operador ferroviario. 
Para el cumplimiento de sus funciones, ADIF puede realizar toda clase de actos de administración y disposición previstos en la legislación civil y mercantil. ADIF no puede prestar servicios de transporte ferroviario, salvo aquellos que sean inherentes a su propia actividad.

Le corresponden a ADIF las siguientes competencias:

a. Aprobación de proyectos básicos y de construcción de infraestructuras ferroviarias que deban formar parte de la Red Ferroviaria de Interés General (RFIG).

b. Construcción de infraestructuras ferroviarias con recursos del Estado o de terceros.

c. Administración de las infraestructuras ferroviarias de su titularidad.

d. Control e inspección de la infraestructura ferroviaria, de sus zonas de protección y de la circulación que sobre ella se produzca.

e. La explotación de los bienes de su titularidad.

f. La elaboración de la Declaración de la Red.

g. La adjudicación de capacidad de infraestructura a las empresas ferroviarias así como celebrar acuerdos marco con ellas.

h. Emisión de informes con carácter previo al otorgamiento de las licencias de empresa ferroviaria y de las autorizaciones para prestar servicios que se hayan declarado de interés público.

i. Elaborar instrucciones y circulares necesarias para determinar las condiciones de operación sobre la Red Ferroviaria de Interés General.

j. Prestación de Servicios Adicionales y, en su caso, Complementarios y Auxiliares, al servicio del transporte ferroviario.

k. Propuesta de tarifas al Ministerio de Fomento por la prestación de Servicios Adicionales y Complementarios y el cobro de las mismas, así como la fijación y cobro de los precios por la prestación de los servicios auxiliares.

1. Propuesta de modificación y actualización de los cánones por utilización de las infraestructuras ferroviarias. Liquidación y recaudación así como el cobro de tarifas por los servicios adicionales, complementarios y auxiliares.

m. Cooperación con organismos que en otros Estados de la Unión Europea administren las infraestructuras ferroviarias.

n. Celebración de acuerdos marco con las empresas ferroviarias.

Establecer pautas reguladoras del procedimiento para realizar la investigación de accidentes ferroviarios que le correspondan y la elaboración de informes anuales que 
contemple todos los incidentes y accidentes producidos como consecuencia de la prestación del servicio de transporte ferroviario.

o. Elaborar un Plan de Contingencias con las medidas para restablecer la situación de normalidad en caso de accidente, fallo técnico o cualquier incidencia que perturbe el tráfico ferroviario.

p. Resolución de reclamaciones de responsabilidad patrimonial a causa de su actividad.

\section{TASAS Y CÁNONES POR USO DE LA INFRAESTRUCTURA FERROVIARIA.}

Las tasas ferroviarias satisfacen los hechos imponibles relacionados con la prestación de servicios ferroviarios regulados en la Ley del Sector Ferroviaria y en la Orden Ministerial correspondiente.

Los cánones se fijan de acuerdo con los principios generales de viabilidad económica de las infraestructuras, explotación eficaz de las mismas, situación del mercado y equilibrio financiero en la prestación de servicios y con criterios de igualdad, transparencia y no discriminación entre prestadores de servicios de transporte ferroviario.

La regulación de cánones ferroviarios que se devengan en favor de ADIF por la utilización de las infraestructuras se establece en la Ley del Sector Ferroviario, fijándose las cuantías resultantes de la aplicación de los elementos y los criterios cuantificadores de los cánones por utilización de las infraestructuras ferroviarias mediante la Orden FOM 898/2005, modificada por la Orden FOM 2336/2012, de 31 de octubre, de conformidad con el Art.77.1 de dicha Ley y a su vez modificada por el R.D. Ley 11/2013 de 2 de agosto, tramitado y aprobado parlamentariamente como Ley 1/2014, de 28 de febrero.

La prestación de los servicios ferroviarios adicionales y complementarios está sujeta al pago de tarifas, que tienen el carácter de precios privados.

El importe de las tarifas se fija atendiendo al tipo de actividad, a su interés ferroviario y a su relevancia económica, así como el coste que suponga la prestación de los servicios.

\subsection{Tasa por licencia y certificado de seguridad.}

Constituye el hecho imponible de la tasa por otorgamiento de la licencia de empresa ferroviaria, la expedición de ésta, su ampliación o su renovación. Así mismo constituye el hecho imponible de la tasa por otorgamiento de certificados de seguridad, de expedición, ampliación, renovación y revisión de los mismos.

\subsection{Tasa por seguridad en el transporte ferroviario de viajeros.}

Constituye el hecho imponible de la tasa por la prestación del servicio de vigilancia y el control de acceso, tanto de viajeros como de equipajes, a las estaciones y demás recintos ferroviarios. Serán sujetos pasivos de la tasa los viajeros.

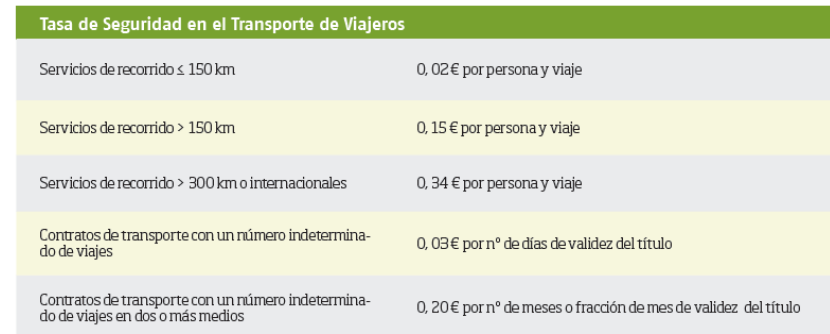

Tabla 1 - Tasa de seguridad en el transporte de viajeros. 


\subsection{Canon por utilización de las infraestructuras ferroviarias.}

El Administrador de Infraestructuras Ferroviarias percibirá por parte de la empresa ferroviaria que utilice la infraestructura de la Red Ferroviaria de Interés General, así como las estaciones y otras instalaciones ferroviarias el abono de las tasas siguientes en concepto de cánones ferroviarios:

- Canon por utilización de las líneas ferroviarias integrantes de la Red Ferroviaria de Interés General. Constituye el hecho imponible del canon la utilización de la línea ferroviaria integrante de la Red Ferroviaria de Interés General así como la prestación de servicios inherentes a dicha utilización.

- Canon de Acceso, por el derecho de utilización con carácter general de la Red Ferroviaria de Interés General o parte de ella. Este canon será en función de los tramos de red en los que se pretenda prestar los servicios y de la declaración de actividad realizada por la empresa, que se pagará de una sola vez, al inicio de cada periodo por el que se haya producido la adjudicación de capacidad.

\begin{tabular}{|llr|}
\hline Nivel & Volumen de Tráfico & $€ /$ Año \\
\hline N1.A & $\leq 0,2$ millones $k m /$ Tren-afio & $60.600,00$ \\
N1.B & $>0,2 y \leq 0,5$ millones de $k m /$ Tren-afio & $151.500,00$ \\
N1.C & $>0,5 y \leq 1$ millones de $k m /$ Tren-afio & $303.000,00$ \\
N2.A & $>1 y \leq 2,5$ millones de $k m /$ Tren-afio & $757.500,00$ \\
N2.B & $>2,5 y \leq 5$ millones de $k m /$ Tren-afio & $1.515 .000,00$ \\
N2.C & $>5 y \leq 10$ millones de $k m /$ Tren-afio & $3.030 .000,00$ \\
N3.A & $>10 y \leq 15$ millones de $k m /$ Tren-afio & $4.545 .000,00$ \\
N3.B & $>15 y \leq 20$ millones de $k m /$ Tren-afio & $6.060 .000,00$ \\
N3.C & $>20 y \leq 30$ millones de $k m /$ Tren-afio & $7.575 .000,00$ \\
N3.D & $>30 y \leq 40$ millones de $k m /$ Tren-afio & $9.090 .000,00$ \\
N3.E & $>40 y \leq 50$ millones de $k m /$ Tren-afio & $12.120 .000,00$ \\
N3.F & $>50$ millones de $k m /$ Tren-año & $15.150 .000,00$ \\
\hline
\end{tabular}

Tabla 2 - Canon de Acceso Líneas Tipo A.

\begin{tabular}{|c|c|c|}
\hline Nivel & Volumen de Tráfico & $€ /$ Año \\
\hline N1A & $\leq 0,2$ millones km/Tren-afio & $13.251,56$ \\
\hline N1.B & $>0,2 \mathrm{y} \leq 0,5$ millones de $\mathrm{km} /$ Tren-año & 33.128,92 \\
\hline N1.C & $>0,5 \mathrm{y} \leq 1$ millones de $\mathrm{km} /$ Tren-afio & $66.257,83$ \\
\hline N2.A & $>1 \mathrm{y} \leq 2.5$ millones de $\mathrm{km} /$ Tren-año & $116.178,60$ \\
\hline N2.B & $>2,5 \mathrm{y} \leq 5$ millones de $\mathrm{km} /$ Tren-afio & $165.644,60$ \\
\hline $\mathrm{N} 2 . \mathrm{C}$ & $>5 \mathrm{y} \leq 10$ millones de $\mathrm{km} /$ Tren-afio & $364.418,11$ \\
\hline N3.A & $>10 \mathrm{y} \leq 15$ millones de km/Tren-año & $761.965,14$ \\
\hline N3.B & $>15 \mathrm{y} \leq 20$ millones de $\mathrm{km} /$ Tren-affo & $1.557 .059,19$ \\
\hline N3.C & $>20 \mathrm{y} \leq 30$ millones de km/Tren-año & $1.557 .059,19$ \\
\hline N3.D & $>30 \mathrm{y} \leq 40$ millones de $\mathrm{km} / \mathrm{Tren}$-afio & $1.557 .059,19$ \\
\hline N3.D & $>40 \mathrm{y} \leq 50$ millones de $\mathrm{km} / \mathrm{Tren}$-afio & $1.557 .059,19$ \\
\hline N3.F & $>50$ millones de km/Tren-afío & $1.557 .059,19$ \\
\hline
\end{tabular}

Tabla 3 - Canon de Acceso Resto de Líneas. 
- Canon por Reserva de Capacidad, en función de los kilómetros de longitud del tramo de red reservado, distinguiendo por tipo de línea y hora del día en que se reserva, y por tipo de servicio de transporte ferroviario y tipo de tren que lo presta.

\begin{tabular}{|c|c|c|c|c|c|c|c|}
\hline \multirow[t]{3}{*}{$\begin{array}{l}\text { Periodo } \\
\text { Horario }\end{array}$} & \multirow[t]{3}{*}{$\begin{array}{l}\text { Tipo } \\
\text { Línea }\end{array}$} & \multicolumn{6}{|c|}{ Tipo de Servicio/Tren } \\
\hline & & VL1 & VL2 & VL3 & VCM & VOT & M \\
\hline & & \multicolumn{6}{|c|}{$€ /$ Tren-km reservado } \\
\hline \multirow{5}{*}{ Punta } & A1 & 2,8371 & 1,5089 & 2,8371 & 1,5089 & 1,5089 & 0.5757 \\
\hline & A2 & 2,7169 & 1,4241 & 2,7169 & 1,4241 & 1,4241 & 0,5757 \\
\hline & $\mathrm{B} 1$ & 1,4241 & 1,4241 & 1.4241 & 1,4241 & 0,4040 & 0,5757 \\
\hline & $\mathrm{C} 1$ & 0,4040 & 0,4040 & 0,4040 & 0,4040 & 0,4040 & 0,3333 \\
\hline & $\mathrm{C} 2$ & 0,4040 & 0,4040 & 0,4040 & 0.4040 & 0,4040 & 0,3333 \\
\hline \multirow{5}{*}{ Normal } & A1 & 2,8371 & 1,5089 & 2,8371 & 1,5089 & 1,5089 & 0,5757 \\
\hline & A2 & 2,7169 & 1,4241 & 2,7169 & 1,4241 & 1,4241 & 0.5757 \\
\hline & B1 & 1,4241 & 1,4241 & 1.4241 & 1,4241 & 0,4040 & 0.5757 \\
\hline & $\mathrm{C} 1$ & 0.4040 & 0.4040 & 0,4040 & 0,4040 & 0,4040 & 0,0505 \\
\hline & C2 & 0,4040 & 0,4040 & 0,4040 & 0,4040 & 0,4040 & 0,0505 \\
\hline \multirow{5}{*}{ Valle } & A1 & 2,8371 & 1,5089 & 2,8371 & 1,5089 & 1,5089 & 0.5757 \\
\hline & A2 & 2,7169 & 1,4241 & 2,7169 & 1,4241 & 1,4241 & 0,5757 \\
\hline & B1 & 1,4241 & 1,4241 & 1.4241 & 1,4241 & 0,4040 & 0,5757 \\
\hline & $\mathrm{C} 1$ & 0,2020 & 0,2020 & 0,2020 & 0,2020 & 0,2020 & 0,0505 \\
\hline & $\mathrm{C} 2$ & 0,2020 & 0,2020 & 0,2020 & 0,2020 & 0,2020 & 0,0505 \\
\hline
\end{tabular}

Tabla 4 - Canon por Reserva de Capacidad.

- Canon de Circulación, en función de los kilómetros de longitud del tramo de red efectivamente utilizado por la empresa, distinguiéndose por tipo de línea y hora del día en que se utiliza, y por tipo de servicio de transporte ferroviario y tipo de tren que lo presta.

\begin{tabular}{ccccccc}
\hline Tipo Línea & \multicolumn{7}{c}{ Tipo de Servicio/ Tren } \\
& VL1 & VL2 & VL3 & VCM & VOT & M \\
\hline & & & $€ /$ Tren-km Circulado & \\
A1 & 2,2018 & 0,8484 & 2,2018 & 0,8484 & 0,8484 & 0,5050 \\
A2 & 2,1008 & 0,7676 & 2,1008 & 0,7676 & 0,7676 & 0,5050 \\
B1 & 0,7676 & 0,7676 & 0,7676 & 0,7676 & 0,1313 & 0,5050 \\
C1 & 0,1212 & 0,1212 & 0,1212 & 0,1212 & 0,1207 & 0,0606 \\
C2 & 0,1212 & 0,1212 & 0,1212 & 0,1212 & 0,1207 & 0,0606 \\
\hline
\end{tabular}

Tabla 5 - Canon de Circulación. 
- Canon por Tráfico, en función del valor económico del servicio de transporte ferroviario prestado, medido en términos de la capacidad ofertada (plazas-km) distinguiendo por hora del día y tipo de línea sobre el que ésta se oferta.

\begin{tabular}{|c|c|c|c|c|c|c|}
\hline \multirow[t]{3}{*}{$\begin{array}{l}\text { Periodo } \\
\text { Horario }\end{array}$} & \multirow[t]{3}{*}{ Tipo Línea } & \multicolumn{5}{|c|}{ Tipo de Servicio/ Tren } \\
\hline & & VL1 & VL2 & VL3 & VCM & VOT \\
\hline & & \multicolumn{5}{|c|}{$€ / 100$ Plazas -km } \\
\hline \multirow{5}{*}{ Punta } & A1 & 2,4019 & 0,7878 & 1,0204 & 1,1358 & 0,0000 \\
\hline & A2 & 1,8617 & 0,7272 & 0,9839 & 0,9055 & 0,0000 \\
\hline & B1 & 0,4833 & 0,4833 & 0,4833 & 0,4833 & 0,0000 \\
\hline & $\mathrm{Cl}$ & 0,0000 & 0,0000 & 0,0000 & 0,0000 & 0,0000 \\
\hline & C2 & 0,0000 & 0,0000 & 0,0000 & 0,0000 & 0,0000 \\
\hline \multirow{5}{*}{ Normal } & Al & 1,6369 & 0,5454 & 0,6955 & 0,7864 & 0,0000 \\
\hline & A2 & 1,2412 & 0,4848 & 0,6560 & 0,6037 & 0,0000 \\
\hline & $\mathrm{B} 1$ & 0,4833 & 0,4833 & 0,4833 & 0,4833 & 0,0000 \\
\hline & $\mathrm{Cl}$ & 0,0000 & 0,0000 & 0,0000 & 0,0000 & 0,0000 \\
\hline & C2 & 0,0000 & 0,0000 & 0,0000 & 0,0000 & 0,0000 \\
\hline \multirow{5}{*}{ Valle } & Al & 1,3922 & 0,4646 & 0,5915 & 0,6698 & 0,0000 \\
\hline & A2 & 1.0085 & 0,3939 & 0,5330 & 0,4905 & 0,0000 \\
\hline & $\mathrm{B} 1$ & 0,4833 & 0,3939 & 0,4833 & 0,4833 & 0,0000 \\
\hline & $\mathrm{Cl}$ & 0,0000 & 0,0000 & 0,0000 & 0,0000 & 0,0000 \\
\hline & $\mathrm{C} 2$ & 0,0000 & 0,0000 & 0,0000 & 0,0000 & 0,0000 \\
\hline
\end{tabular}

Tabla 6 - Canon por Tráfico.

2.4 Canon por utilización de las estaciones y otras instalaciones ferroviarias. Constituye el hecho imponible del canon la utilización de las estaciones y otras instalaciones ferroviarias integrantes de la Red Ferroviaria de Interés General, así como la prestación de servicios inherentes a dicha utilización.

- Canon por utilización de estaciones por parte de los viajeros, la duración del recorrido del transporte y la categoría de la estación.

\begin{tabular}{|c|c|c|c|c|c|c|}
\hline \multicolumn{2}{|l|}{ Tipo de Trayecto } & \multirow[t]{3}{*}{ Categoría } & \multicolumn{4}{|c|}{ Duración del Recorrido/ Trayecto } \\
\hline \multirow{2}{*}{ A } & \multirow{2}{*}{ Superior a 250 km } & & A & B & c & D \\
\hline & & & \multicolumn{4}{|c|}{$€ /$ Viajero } \\
\hline B & Entre 126 y $250 \mathrm{~km}$ & $1^{\mathrm{a}}$ & 1,3519 & 0.5450 & 0.2369 & 0.0824 \\
\hline C & Entre 80 y $125 \mathrm{~km}$ & $2^{\mathrm{a}}$ & 0.6269 & 0,3910 & 0,1778 & 0.0618 \\
\hline D & Inferior a $80 \mathrm{~km}$ & $3^{\mathrm{a}}$ & 0,0474 & 0.0474 & 0,0474 & 0.0206 \\
\hline
\end{tabular}

Tabla 7 - Canon por Utilización de Estaciones por viajeros 
- Canon por el estacionamiento y la utilización de andenes en las estaciones, el tiempo de estacionamiento del tren, realización de operaciones de cambio de vía a solicitud del operador y categoría de la estación.

\begin{tabular}{|c|c|c|c|c|c|}
\hline \multicolumn{2}{|c|}{ Tipo de Estacionamiento } & \multirow[t]{3}{*}{ Categoría } & \multicolumn{3}{|c|}{ Estacionamiento } \\
\hline \multirow{2}{*}{ A } & \multirow{2}{*}{ Por cada 5 minutos adicionales o fracción entre $15 \mathrm{~min}$ y $45 \mathrm{~min}$. } & & A & B & c \\
\hline & & & \multicolumn{3}{|c|}{$€ /$ Tren } \\
\hline \multirow{2}{*}{ B } & \multirow{2}{*}{ Por cada 5 minutos adicionales o fracción entre $45 \mathrm{~min}$ y $120 \mathrm{~min}$. } & $1^{\mathrm{a}}$ & 2,2458 & 3,3688 & 4,4917 \\
\hline & & $2^{\mathrm{a}}$ & 1,1229 & 1,6998 & 2.2458 \\
\hline C & Por cada 5 minutos adicionales o fracción a partir de los 120 min. & $3^{\mathrm{a}}$ & - & - & - \\
\hline
\end{tabular}

Tabla 8 - Canon por Estacionamiento y Utilización de Andenes.

- Canon por paso por cambiadores de ancho.

Valor unitario por tren

$112,64 €$

Tabla 9 - Canon por Paso por Cambiadores de Ancho.

- Canon por utilización de vías de apartado, el tiempo de ocupación de la vía, tipo de tren y tipo de línea.

\begin{tabular}{|c|c|c|c|c|c|c|c|}
\hline \multicolumn{2}{|c|}{ Tipo de Estacionamiento } & \multirow[t]{2}{*}{ Tipo de Línea } & \multirow[t]{2}{*}{ Tipo de Servicio / Tren } & \multicolumn{4}{|c|}{ Estacionamiento } \\
\hline a & Entre 1 y 6 horas. & & & $a$ & b & c & d \\
\hline $\mathrm{b}$ & Por cada hora de estacionamiento desde la hora sexta hasta la decimosegunda. & & & \multicolumn{4}{|c|}{$€ /$ Tren } \\
\hline c & Por cada hora de estacionamiento a partir de la decimosegunda hora. & A & VL1-VL2-VL3-VCM & 16,2669 & 2,1428 & 3,1627 & 40,5487 \\
\hline d & Estacionamiento por día completo. & B-C & - & - & - & - & - \\
\hline
\end{tabular}

Tabla 10 - Canon por Utilización de Vías de Apartado.

- Canon por la prestación de servicios que precisen de autorización para la utilización del dominio público ferroviario.

$$
\text { Zona de dominio público } \quad 0,6799 € / \mathrm{m}^{2} \text {-mes }
$$

Tabla 11 - Canon por Utilización del Dominio Público Ferroviario.

2.5 Tarifa por Servicios Complementarios, la prestación de los Servicios

Complementarios estará sujeta al pago de Tarifas, que tendrán el carácter de precios privados. Las tarifas de estos servicios prestados serán aprobadas por el explotador de la instalación de servicio, previo informe de la Comisión Nacional de los Mercados y la Competencia.

- Manipulación de Unidades de Transporte Intermodal.

- Suministro de la Corriente de Tracción.

- Suministro de Combustible.

- Transportes Excepcionales. 


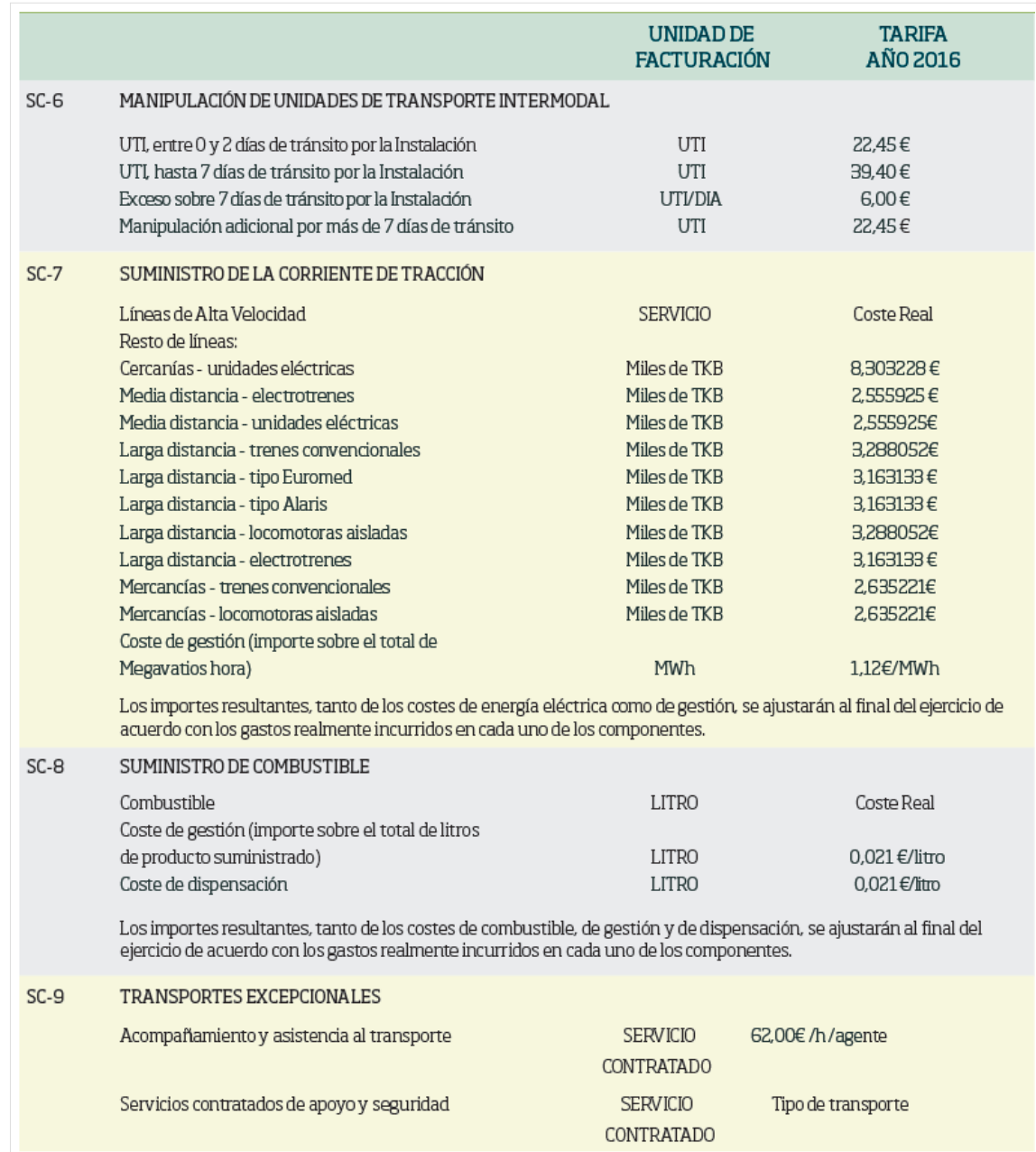

Tabla 12 - Tarifas por Servicios Complementarios.

\section{REFERENCIAS.}

ADIF Dirección General de Servicios a Clientes y Patrimonio. Declaración sobre la Red 2016 ,

FONT TORRES, J.B. (2016), Explotación y Gestión por Operador Privado Línea de Alta Velocidad Madrid-Valencia. Trabajo Final de Máster.

MINISTERIO DE FOMENTO. Gobierno de España, Informe de la Comisión Técnica para el estudio de mejoras en el sector ferroviario.

FUNDACIÓN DE LOS FERROCARRILES ESPAÑOLES. Observatorio del Ferrocarril en España.

Reglamento (CE) 881/2004 del Parlamento Europeo y del Consejo, de 29 de abril de 2004 Por el que se crea una Agencia Ferroviaria Europea.

DIARIO OFICIAL DE LA UNIÓN EUROPEA L 164, de 30 de abril de 2004. Modificado por: REGLAMENTO (CE) No 1335/2008 DEL PARLAMENTO EUROPEO Y DEL CONSEJO de 16 de diciembre de 2008.

Rectificado por: DO L 220, 21.6.2004, p. 3 (881/04). Anexo 131 
Reglamento (CE) 653/2007 de la Comisión, de 13 de junio de 2007.

Sobre el uso de un formato europeo común para los certificados de seguridad y los documentos de solicitud, de conformidad con el Art. 10 de la Directiva 2001/49/CE del Parlamento Europeo y del Consejo, y sobre la validez de los certificados de seguridad expedidos en virtud de la Directiva 2001/14/CE del Parlamento Europeo y del Consejo. DIARIO OFICIAL DE LA UNIÓN EUROPEA L 153, de 14 de junio de 2007.

Modificado por: Reglamento (UE) no 445/2011 de la Comisión de 10 de mayo de 2011.

Reglamento (UE) No 1158/2010 de la Comisión, de 9 de diciembre de 2010.

Sobre un método común de seguridad para evaluar la conformidad con los requisitos para la obtención de un certificado de seguridad ferroviaria.

DIARIO OFICIAL DE LA UNIÓN EUROPEA, de 10 de diciembre de 2010.

Rectificado: DO L 032, 8.2.2011, p. 23 (1158/2010).

Reglamento (UE) No 1169/2010 de la Comisión, de 10 de diciembre de 2010.

Sobre un método común de seguridad para evaluar la conformidad con los requisitos para la obtención de una autorización de seguridad ferroviaria.

DIARIO OFICIAL DE LA UNIÓN EUROPEA, de 11 de diciembre de 2010.

Reglamento (UE) No 1077/2012 de la Comisión, de 16 noviembre de 2012.

Relativo a un método común de seguridad para la supervisión por parte de las autoridades nacionales de seguridad tras la expedición de certificado de seguridad o autorizaciones de seguridad.

DIARIO OFICIAL DE LA UNIÓN EUROPEA de 17 de noviembre de 2012.

Directiva 2004/49/CE del Parlamento Europeo y del Consejo, de 29 de abril de 2004.

Sobre la seguridad de los ferrocarriles comunitarios y por la que se modifican la Directiva 95/18/CE del Consejo sobre concesión de licencias a las Empresas Ferroviarias y la Directiva 2001/14/CE relativa a la Adjudicación de la Capacidad de Infraestructura Ferroviaria, aplicación de cánones por su utilización y certificación de la seguridad.

DIARIO OFICIAL DE LA UNIÓN EUROPEA, L 164 de 30 de abril de 2004, L 220 de 21de junio de 2004, L313/65 de 28 de noviembre de 2009.

Modificado por: DIRECTIVA 2008/57/CE DEL PARLAMENTO EUROPEO Y DEL CONSEJO Texto pertinente a efectos del EEE de 17 de junio de 2008., DIRECTIVA 2008/110/CE DEL PARLAMENTO EUROPEO Y DEL CONSEJO Texto pertinente a efectos del EEE de 16 de diciembre de 2008, DIRECTIVA 2009/149/CE DE LA COMISIÓN Texto pertinente a efectos del EEE de 27 de noviembre de 2009. DIRECTIVA 2014/88/UE DE LA COMISIÓN Texto pertinente a efectos del EEE de 9 de julio de 2014. Rectificado por: DO L 220, 21.6.2004, p. 16 (2004/49) Anexo 133.

Ley 39/2003, de 17 de noviembre, del Sector Ferroviario.

BOE de 18 de noviembre de 2003.

SE AÑADE la disposición adicional 14, por Ley 18/2014, de 15 de octubre (Ref. BOE-A2014-10517).

SE MODIFICA los arts. 77 y 81.1.j), por Ley 15/2014, de 16 de septiembre (Ref. BOE-A2014-9467).

SE AÑADE la disposición adicional 14, por Real Decreto-ley 8/2014, de 4 de julio (Ref. BOE-A-2014-7064).

SE MODIFICA:

Los arts. 21, 73, 77, 81, 89, 90 y disposición transitoria 3 y SE AÑADE las disposiciones 
adicionales 11 a 13 y transitoria 8, por Ley 1/2014, de 28 de febrero (Ref. BOE-A-20142219).

Arts. 56, 57, 58, 60, 63, 71, 77 y 81 y SE AÑADE los arts. 56 bis, 57 bis y disposiciones adicional 13 y transitoria 8 .

Real Decreto-ley 1/2014, de 24 de enero (Ref. BOE-A-2014-747).

SE MODIFICA:

los arts. 20 y 22, por Real Decreto-ley 15/2013, de 13 de diciembre (Ref. BOE-A-201313035).

los arts. 21.1), 73, 77, 81.1.j) y SE AÑADE la disposición adicional 12, por Real Decretoley 11/2013, de 2 de agosto (Ref.BOE-A-2013-8556).

las disposiciones adicional 9.1 y transitoria 3, por Ley 11/2013, de 26 de julio (Ref. BOEA-2013-8187).

SE DEROGA arts. 82 a 84 y SE MODIFICA los arts. 95 y 96, por Ley 3/2013, de 4 de junio (Ref. BOE-A-2013-5940).

SE MODIFICA las disposiciones adicional 9.1 y transitoria 3, por Real Decreto-ley 4/2013, de 22 de febrero (Ref. BOE-2013-2030).

SE DECLARA en los RECURSOS, acumulados, 908/2004, 909/2004, 917/2004, 918/2004, 930/2004 y 933/2004 (BOEA- 2004-5150, BOE-A-2004-5151, BOE-A-2004-5152, BOEA-2004-5153, BOE-A-2004-5154 y BOE-A-2004-5155),la inconstitucionalidad y nulidad de la disposición adicional 9.1 y la DESESTIMACIÓN de todo lo demás, por Sentencia 245/2012, de 18 de diciembre (Ref. BOE-A-2013-618).

SE DEROGA en la forma indicada la disposición transitoria 5, por Ley 17/2012, de 27 de diciembre (Ref. BOE-A-2012-15651).

SE MODIFICA:

la disposición transitoria 3, por Decreto Ley 22/2012, de 20 de julio (Ref. BOE-A-20129772).

los arts. 8, 40 y 82 a 84, por Ley 2/2011, de 4 de marzo (Ref. BOE-A-2011-4117).

SE MODIFICA:

los arts. 21.1, 40, 41, 78, 79.1, el anexo y AÑADE la disposición transitoria 7, por Ley 25/2009, de 22 de diciembre (Ref. BOE-A-2009-20725).

los arts. 90.2.e) y disposición transitoria 2 y SE AÑADE la adicional 10, por Ley 15/2009, de 11 de noviembre (Ref.BOE-A-2009-18004).

el art. 36.2, con efectos de 1 de noviembre de 2007, por Ley 31/2007, de 30 de octubre (Ref. BOE-A-2007-18875).

el art. 22.3.b), con efectos de 30 de abril de 2008, por Ley 30/2007, de 30 de octubre (Ref. BOE-A-2007-18874).

SE MODIFICA el art. 6, por Ley 30/2005, de 29 de diciembre (Ref. BOE-A-2005-21525).

SE MODIFICA los arts. 65 y 66, por Ley 4/2004, de 29 de diciembre (Ref. BOE-A-200421831).

SE AMPLIA el plazo de la disposición final 3.1, por Real Decreto Ley 1/2004, de 7 de mayo (Ref. BOE-A-2004-8765).

Real Decreto 2387/2004, de 30 de diciembre.

Por el que se aprueba el Reglamento del Sector Ferroviario.

BOE de 31 de diciembre de 2004.

SE MODIFICA la disposición adicional 11, por Real Decreto 623/2014, de 18 de julio (Ref. BOE-A-2014-7651).

SE DEROGA el título VI, por Real Decreto 657/2013, de 30 de agosto (Ref. BOE-A-20139212).

SE MODIFICA el art. 56, por Real Decreto 641/2011, de 9 de mayo (Ref. BOE-A-2011- 
8124).

SE MODIFICA:

los arts. 129 y 134, por Real Decreto 1434/2010, de 5 de noviembre (Ref. BOE-A-201017037).

los arts. 54 a 56 y 78.2.f) y SE SUPRIME la disposición adicional 10, por Real Decreto 100/2010, de 5 de febrero (Ref.BOE-A-2010-3749).

SE DEROGA los capítulos V y VI del título III y SE MODIFICA los arts. 16.1, 27.2, 35.2, 63.2 y $3,82,88,133,134.2$ y el anexo,

por Real Decreto 810/2007, de 22 de junio (Ref. BOE-A-2007-13177).

SE DEROGA la disposición adicional 14 y SE MODIFICAN determinados preceptos, por Real Decreto 354/2006, de 29 de marzo (Ref. BOE-A-2006-6246).

Real Decreto 2395/2004, de 30 de diciembre.

Por el que se aprueba el Estatuto de la Entidad Pública Empresarial Administrador de Infraestructuras Ferroviarias.

BOE de 31 de diciembre de 2004.

SE MODIFICA los arts. 1, 3, 4, 6, 9, 11, 13, 16, 17, 23, 27, 30, 31, 33, 34 y 40, por Real Decreto 1044/2013, de 27 de diciembre (Ref. BOE-A-2013-13727).

SE MODIFICA:

el art. 15.1, por Real Decreto 104/2011, de 28 de enero (Ref. BOE-A-2011-3586).

los arts. 3.1 y 16.1.p), por Real Decreto 458/2010, de 16 de abril (Ref. BOE-A-2010-6755). CORRECCIÓN de errores en BOE núm. 23 de 27 de enero de 2005 (Ref. BOE-A-20051360 .

Real Decreto 1044/2013, de 27 de diciembre.

Por el que se aprueba el Estatuto de la Entidad Pública Empresarial ADIF-Alta Velocidad. BOE de 28 de diciembre de 2013.

Orden FOM/897/2005, de 7 de abril.

Relativa a la Declaración sobre la Red y al procedimiento de Adjudicación de Capacidad de Infraestructura Ferroviaria. BOE de 9 de abril de 2005.

SE MODIFICA: Orden FOM/420/2014, de 7 de marzo, BOE de 19 de marzo de 2014.

Orden FOM/898/2005, de 8 de abril.

Por la que se fijan las cuantías de los cánones ferroviarios establecidos en los artículos 74 y 75 de la Ley 39/2003, de 17 de noviembre, del Sector Ferroviario. BOE de 9 de abril de 2005.

\section{SE MODIFICA:}

el art. 1 y los anexos I, II y III, por Ley 1/2014, de 28 de febrero (Ref. BOE-A-2014-2219). los arts. 1 y 2, por Ley 22/2013, de 23 de diciembre (Ref. BOE-A-2013-13616).

el art. 1.1.a) y d) y los anexos I a III, por Real Decreto-ley 11/2013, de 2 de agosto (Ref. BOE-A-2013-8556).

los arts. 1 y 2 y los anexos I, II, IV y V, por Orden FOM/2336/2012, de 31 de octubre (Ref. BOE-A-2012-13530).

Orden FOM/3236/2010, de 13 de diciembre, BOE 15 diciembre 2010.

los anexos II y V, por Orden FOM/3417/2011, de 1 de diciembre (Ref. BOE-A-2011-19653). los anexos II Y V, por Orden FOM/3852/2007, de 20 de diciembre (Ref. BOE-A-200722444). 
Orden FOM/2257/2010, de 2 de agosto.

Por la que se establece la fecha a partir de la que la Dirección General de Infraestructuras Ferroviarias asumirá las competencias en materia de certificados de seguridad establecidas en el Reglamento sobre Seguridad en la Circulación de la Red Ferroviaria de Interés General. BOE de 23 de agosto de 2010.

Orden FOM/189/2015, de 11 de febrero.

Por la que se desarrollan principios básicos de aplicación de incentivos en el sistema de los cánones por utilización de las infraestructuras ferroviarias, establecidas en el Art.73 de la Ley 39/2003, de 17 de noviembre, del Sector Ferroviario. BOE de 12 de febrero de 2015.

Orden FOM/710/2015, de 30 de enero.

Por la que se aprueba el Catálogo de líneas y tramos de la Red Ferroviaria de Interés General BOE de 23 de abril de 2015. 